\title{
Radial Shortening Osteotomy for Symptomatic Kienböck's Disease: Complications and Long-Term Patient-Reported Outcome
}

\author{
Wouter F. van Leeuwen, MD ${ }^{1,2}$ Taylor M. Pong, BSc ${ }^{1}$ Rachel W. Gottlieb, BSc ${ }^{1}$ \\ Christian Deml, MD ${ }^{1,3}$ Neal Chen, MD ${ }^{1}$ Brigitte E.P.A. van der Heijden, MD, PhD ${ }^{2,4}$ \\ ${ }^{1}$ Hand and Upper Extremity Service, Department of Orthopaedic \\ Surgery, Massachusetts General Hospital, Harvard University \\ Medical School, Boston, Massachusetts \\ 2 Department of Plastic, Reconstructive and Hand Surgery, \\ Jeroen Bosch Hospital, 's-Hertogenbosch, The Netherlands \\ ${ }^{3}$ Department of Trauma Surgery, Medical University of Innsbruck, \\ Innsbruck, Austria \\ ${ }^{4}$ Department of Plastic and Reconstructive Surgery, Radboud \\ University Medical Center, Nijmegen, The Netherlands \\ J Wrist Surg 2021;10:17-22.
}

\section{Abstract}

\section{Keywords}

- Kienböck's disease

- lunatomalacia

- radial shortening osteotomy

- vascularized bone graft

- patient-reported outcome

- long-term follow-up
Objective To determine the rate of salvage procedures and any other unplanned reoperations in patients with symptomatic Kienböck's disease who were treated with radial shortening osteotomy. In addition, we studied patient-reported outcome in the long term using Patient-Reported Outcome Measure Information System (PROMIS) instruments.

Patients and Methods We performed a retrospective review of all patients who underwent radial shortening osteotomy for stage 2 and 3A Kienböck's disease. Patients who had concomitant revascularization were grouped separately. We collected demographic data, data regarding type of surgery and reoperations, and radiographic data. Patient-reported outcome measures were the PROMIS Upper Extremity Computer Adaptive Testing (CAT) and Pain Interference instruments, the abbreviated Disabilities of Arm, Shoulder, and Hand (QuickDASH), and the 0 to 10 numeric rating scale for pain and satisfaction.

Results We included 48 patients who had radial shortening osteotomy alone, and 17 patients who had a combined procedure of radial shortening and direct revascularization. The rate of unplanned reoperations was 33\% (16 of 48) in those who had radial shortening osteotomy and $24 \%$ ( 4 of 17 ) in those who had a combined procedure. Six (13\%) of 48 patients underwent proximal row carpectomy due to failed radial shortening osteotomy. No salvage procedures were performed after combined radial shortening/revascularization. Median PROMIS Physical Function CT scores were 56 (interquartile range [IQR]: 44-56) and 56 (IQR: 41-56), respectively. Median PROMIS Pain Interference scores were 39 (IQR: 39-52) and 39 (IQR: 39-49), respectively. Median QuickDASH scores were 2.3 (IQR: 0-23) and 4.5 (IQR: 2.3-14), respectively.

Conclusion Radial shortening osteotomy for symptomatic Kienböck's disease yields reasonable long-term function. We observed that approximately one in eight patients received

February 22, 2020

accepted

June 17,2020

published online

August 20, 2020 (c) 2020. Thieme. All rights reserved.

Thieme Medical Publishers, Inc.,

333 Seventh Avenue, 18th Floor,

New York, NY 10001, USA
DOI https://doi.org/

10.1055/s-0040-1714750.

ISSN 2163-3916. 
underwent salvage surgery after radial shortening, and this should be taken into account when making the initial decision to treat Kienböck's disease surgically. There appeared to be no benefit of direct revascularization in addition to radial shortening in terms of patient-reported outcome in the long term.

Level of Evidence This is a Level IV, therapeutic study.

The choice of surgery for symptomatic Kienböck's disease depends mainly on radiographic factors such as disease stage $^{1}$ and ulna variance, ${ }^{2}$ as well as patient and surgeon preference. ${ }^{3,4}$ Radial shortening osteotomy is a widely accepted procedure for Kienböck's disease without secondary changes in carpal alignment or radiocarpal osteoarthritis (e.g., stage 2 and $3 \mathrm{~A}$ according to the Lichtman classification) and negative or neutral ulna variance. ${ }^{5}$ Radial shortening osteotomy levels the distal surfaces of the radius and ulna, redistributes the axial load across the carpus, and subsequently decreases mechanical pressure on the lunate. ${ }^{6}$ In contrast, insertion of vascularized bone or arteriovenous pedicle grafts into the lunate aims to induce neovascularization and new bone formation in the lunate.

It is hoped that surgery can halt disease progression, decrease pain, and improve function. Some patients do not respond well enough to surgery and are offered salvage surgery, such as proximal row carpectomy or arthrodesis, due to progressive pain and carpal collapse. ${ }^{7,8}$ It is unclear how often patients have salvage surgery after initial radial shortening osteotomy. Furthermore, long-term patientreported outcome data are scarce.

The aim of this study was to determine the rate of salvage surgery for failed initial treatment and any other unplanned reoperations after radial shortening osteotomy for symptomatic Kienböck's disease. Secondarily, we studied the patient-reported outcome in the long term using PatientReported Outcome Measure Information System (PROMIS) instruments. Finally, we studied patient-reported outcome using the more commonly used abbreviated Disabilities of Arm, Shoulder, and Hand (QuickDASH) and numeric rating scale (NRS) for pain and satisfaction.

\section{Patients and Methods}

Our Institutional Review Board approved the protocol for this retrospective study. We identified all patients who had a radial shortening osteotomy for symptomatic Kienböck's disease between January 1992 and December 2013 from our institutional database and performed medical record and radiograph review. We included patients who were at least 18 years old at the time of the study. We excluded patients who were skeletally immature at the time of surgery $(n=1)$, had prior surgery for their Kienböck's disease $(n=6)$, had Lichtman stage $1(n=2)$, or had a radioscaphoid angle of more than 60 degrees on preoperative wrist radiographs, representing Lichtman stage $3 \mathrm{~B}(n=4))^{9,10}$

Our study population consisted of 65 patients, of whom 48 had a radial shortening osteotomy and 17 had a combined procedure of radial shortening osteotomy and direct revascularization. The average age at the time of surgery was 37 years (interquartile range [IQR]: $29-44$ years; range: $17-57$ years). Thirty-seven patients (57\%) were men. Hand dominance was recorded for $47 / 65$ patients, and the dominant side was affected in $66 \%$. In all patients, the diagnosis was made based on clinical and radiographic findings. In 13 patients, magnetic resonance imaging (MRI) scans were obtained by our institution. Some patients had outside advanced imaging. The preoperative Lichtman stages were 2 in 23 patients and $3 \mathrm{~A}$ in 42 patients. All patients had undergone a trial of immobilization for at least 6 to 12 weeks prior to surgery.

The radial shortening osteotomy was performed using a volar approach and plating in all patients. An osteotomy was created using an oscillating saw, and, on average, $2 \mathrm{~mm}$ (IQR: 1-3 $\mathrm{mm}$; range: $1-5 \mathrm{~mm}$ ) of bone was resected. Internal fixation was obtained by means of a (limited contact) dynamic compression plate, T-plate, or RAYHACK system (Wright Medical Group). The median postoperative ulna variance amounted to -0.8 (IQR: -1.9 to 0.45 ) millimeter. Of the 17 patients (26\%) who had a concomitant revascularization procedure, 13 patients had a pedicled vascularized bone graft from the distal radius to the lunate based on either the third and fourth $(n=4)$ or fourth and fifth $(n=9)$ extensor compartmental arteries, and 4 patients had implantation of the superficial branch of the radial artery and vein into the lunate, packed with free cancellous bone from the distal radius. Four patients had a wrist arthroscopy with synovectomy, seven patients had excision of both the anterior and posterior interosseous nerves or the posterior interosseous nerve alone, two patients had open carpal tunnel release, one patient had resection of a giant cell tumor of the thumb, and one patient had submuscular transposition of the ulnar nerve at the elbow performed in the same surgery. Postoperatively, a splint was applied for approximately 4 weeks in all patients. The procedures were performed by 11 attending orthopedic hand surgeons at two academic medical centers and one affiliated community hospital.

We reached out to all 65 patients by mail and telephone to invite them for questionnaire follow-up. Of the 65 patients, 24 did not respond or had incorrect contact information, 5 declined to participate, 4 agreed but never filled out the questionnaires, 2 did not speak English, and 2 had died of circumstances unrelated to Kienböck's disease. The 28 patients whose patient-reported outcomes were collected resembled the remaining 37, who were only available for medical record review, in terms of age, sex distribution, preoperative Lichtman stage, and additional surgeries $(p>0.05)$. 


\section{Study Variables and Patient-Reported Outcome Measures}

The primary outcome measures were subsequent salvage procedures and any other unplanned reoperations related to the radial shortening osteotomy or Kienböck's disease. Secondary, we studied patient-reported outcomes. The main patientreported outcome measure was the PROMIS Physical Function Upper Extremity Computer Adaptive Testing (CAT) instrument. Computerized adaptive testing optimizes the questionnaire administration by distributing only relevant items based on previous responses, shortens completion time, and decreases floor and ceiling effects. ${ }^{11}$ The PROMIS Upper Extremity CAT measures patient-reported disability and results in a standardized $t$-score with a mean of 50 and a standard deviation (SD) of 10. A lower $t$-score means more disability. We prefer the PROMIS instruments because of their efficiency and precision. ${ }^{11}$

In addition, we administered the PROMIS Pain Interference instrument to measure self-reported consequences of pain on social, cognitive, emotional, physical, and recreational aspects of life. A higher score means more impediment in daily life due to pain. We also administered the QuickDASH questionnaire, which uses 11 items to measure physical function and symptoms in patients with upper extremity disorders. The QuickDASH results in a score between 0 and 100, and a higher score represents more disability. Finally, patients were asked to rate their current pain as well as their satisfaction with the surgery on a 0 to 10 NRS, and whether they would elect to have the same surgery again. Patient-reported outcome data were collected using RedCap (Research Electronic Data Capture, Vanderbilt University, Nashville, TN).

We gathered information on the following explanatory variables: age at the time of surgery, sex, affected side and hand dominance, initial Lichtman stage, date and type of surgical procedure(s), and concomitant procedures. The ulna variance in relation to the midpoint between the volar and dorsal lips of the distal radius was measured on postoperative radiographs using the method of perpendiculars. ${ }^{12}$

\section{Statistical Analysis}

We reported categorical data as absolute numbers and percentages, and we used the Fisher exact test to compare the proportions of categorical variables between two groups. We reported continuous data as median and interquartile range (IQR) because most data were nonnormally distributed. A Mann-Whitney $U$ test was used to compare the median of continuous variables between two groups. Statistical analyses were performed using Stata 14 (StataCorp., College Station, TX), and a $p$-value of less than 0.05 was considered statistically significant.

\section{Results}

We stratified the 65 patients into those who had a radial shortening osteotomy alone $(n=48)$ and those who had a radial shortening combined with a revascularization procedure $(n=17 ;$ - Table 1$)$. Of the former 48 patients, $16(33 \%)$ had an additional unplanned surgery ( - Table 1). In six (13\%) patients, radial shortening had failed to resolve the symptoms and, subsequently, a proximal row carpectomy was performed after a median of 18 months (IQR: 7-55 months; range: 7 months to 9.3 years). Four of those patients had preoperative Lichtman stage 2 , whereas two patients had stage $3 \mathrm{~A}$ preoperatively $(p=0.17)$. Two $(4.2 \%)$ other patients developed symptoms of ulnocarpal impaction after surgery and subsequently had an ulna shortening osteotomy. A total of seven $(15 \%)$ patients had removal of the symptomatic plate and screws, and for two of those it was the only reoperation.

Of 17 patients, 4 (24\%) had an additional surgery after combined radial shortening/vascularized bone grafting (-Table 1). None of these patients required salvage surgery. All four patients had removal of symptomatic hardware, of whom one patient also had arthroscopic debridement of the wrist. - Table 2 provides an overview of all unplanned reoperations. The rates of reoperation were comparable for both groups ( $p=0.56$; -Table 1$)$.

Table 1 Patient characteristics $(n=65)$

\begin{tabular}{|c|c|c|c|c|c|}
\hline & \multicolumn{2}{|l|}{$\begin{array}{l}\text { Radial shortening } \\
\text { osteotomy }(n=48)\end{array}$} & \multicolumn{2}{|c|}{$\begin{array}{l}\text { Combined radial shortening } \\
\text { and VBG }(n=17)\end{array}$} & \multirow[t]{2}{*}{$p$-Value } \\
\hline & Median or number & IQR or $\%$ & Median or number & IQR or \% & \\
\hline Age, median (IQR) & 35 & $27-42$ & 44 & $31-51$ & 0.025 \\
\hline Men, $n(\%)$ & $31 / 48$ & $65 \%$ & $6 / 17$ & $35 \%$ & 0.048 \\
\hline \multicolumn{6}{|l|}{ Preoperative Lichtman } \\
\hline Stage $2, n(\%)$ & $19 / 48$ & $40 \%$ & $4 / 17$ & $24 \%$ & \multirow[t]{2}{*}{0.38} \\
\hline Stage $3 \mathrm{~A}, n(\%)$ & $29 / 48$ & $60 \%$ & $13 / 17$ & $76 \%$ & \\
\hline $\begin{array}{l}\text { Postoperative ulna variance } \\
\text { (in millimeter), median (IQR) }\end{array}$ & -0.8 & -1.8 to 0.3 & -0.1 & -2.6 to 0.6 & 0.75 \\
\hline Reoperation, $n(\%)$ & $16 / 48$ & $33 \%$ & $4 / 17$ & $24 \%$ & 0.55 \\
\hline Salvage surgery, $n(\%)$ & $6 / 48$ & $13 \%$ & $0 / 17$ & $0.0 \%$ & 0.33 \\
\hline Follow-up, $n(\%)$ & $14 / 48$ & $29 \%$ & $11 / 17$ & $65 \%$ & 0.019 \\
\hline
\end{tabular}

Abbreviations: IQR, interquartile range, VBG, vascularized bone graft.

Note: Values in bold are statistically significant $(p<0.05)$. 
Table 2 Overview of all unplanned reoperations

\begin{tabular}{|l|l|l|}
\hline Type of reoperation & $\begin{array}{l}\text { Radial shortening, } \\
\boldsymbol{n}(\%)\end{array}$ & $\begin{array}{l}\text { Radial shortening } \\
\text { with VBG, } \boldsymbol{n}(\%)\end{array}$ \\
\hline Proximal row carpectomy & $6 / 48(13 \%)$ & $0 / 17$ \\
\hline Hardware removal only & $2 / 48(4.2 \%)^{\mathrm{a}}$ & $3 / 17(18 \%)^{\mathrm{b}}$ \\
\hline Arthroscopic debridement & $3 / 48(6.3 \%)$ & $1 / 17(5.9 \%)$ \\
\hline $\begin{array}{l}\text { Ulna shortening osteotomy due } \\
\text { to new-onset ulnar-sided wrist pain }\end{array}$ & $2 / 48(4.2 \%)$ & $0 / 17$ \\
\hline Revision radial shortening due to loose hardware & $1 / 48(2.1 \%)$ & $0 / 17$ \\
\hline Repair of radius nonunion with callus autograft & $1 / 48(2.1 \%)$ & $0 / 17$ \\
\hline $\begin{array}{l}\text { Repair of ruptured extensor pollicis longus } \\
\text { tendon due to prominent hardware }\end{array}$ & $1 / 48(2.1 \%)$ & $0 / 17$ \\
\hline
\end{tabular}

Abbreviation: VBG, vascularized bone grafting.

${ }^{a}$ Five more patients had hardware removal after or during proximal row carpectomy $(n=2)$, arthroscopic debridement $(n=2)$, and nonunion repair $(n=1)$.

${ }^{\mathrm{b}}$ One more patient eventually had hardware removal after initial arthroscopic debridement.

Excluding the six patients who underwent an additional salvage procedure, patient-reported outcomes were available for 25 of 59 patients, corresponding to a response rate of $42 \%$. The median time between the primary surgery and questionnaire follow-up was 13 years (IQR: 9-14 years; range: 4-25 years). Some patients had additional procedures in the meantime (-Table 2). - Table 3 summarizes and compares the average patient-reported outcomes for patients who had radial shortening alone $(n=14)$ and those who had combined radial shortening/revascularization procedures $(n=11)$. We observed no notable differences in any of the patient-reported outcomes between both groups. One patient indicated that she would not elect to have a radial shortening osteotomy again. There were no differences in whether the dominant or nondominant side was affected for any of the outcomes in both groups $(p>0.05)$.

\section{Posthoc Power Analysis}

A posthoc power analysis demonstrated that the two groups of 14 and 11 patients provided $80 \%$ statistical power (twotailed $\alpha: 0.05$ ) to detect a difference in average PROMIS Upper Extremity CAT score, with a large effect size of 1.18 using a parametric test. The PROMIS instruments are designed in order that $1 \mathrm{SD}$ is equivalent to 10 points. In other words, we would have been able to detect a difference in PROMIS Upper Extremity CT score of 12 points or more between the groups, with $80 \%$ statistical power using a parametric test. Considering that the average minimal clinically important difference for the PROMIS Upper Extremity CAT is reported to be 9.0 in various hand conditions, ${ }^{13}$ we would have been able to detect such a clinically relevant difference with $57 \%$ statistical power using a parametric test.

Table 3 Characteristics and patient-reported outcome of patients who were available for follow-up $(n=25)$

\begin{tabular}{|l|l|l|l|l|l|}
\hline & \multicolumn{2}{|l|}{$\begin{array}{l}\text { Radial shortening osteotomy } \\
(\boldsymbol{n}=\mathbf{1 4})\end{array}$} & \multicolumn{2}{l}{$\begin{array}{l}\text { Combined radial shortening and } \\
\text { VBG }(\boldsymbol{n}=\mathbf{1 1})\end{array}$} & \multirow{2}{*}{-Value } \\
\hline & Median or number & IQR or \% & Median or number & IQR or \% & \\
\hline Age, median (IQR) & 40 & $29-42$ & 41 & $31-51$ & 0.35 \\
\hline Men, $\boldsymbol{n}$ (\%) & $8 / 14$ & $57 \%$ & $4 / 11$ & $36 \%$ & 0.43 \\
\hline Preoperative Lichtman & & & & & \\
\hline Stage 2, $n$ (\%) & $4 / 14$ & $29 \%$ & $4 / 11$ & $36 \%$ & $>0.99$ \\
\hline Stage 3A, $n$ (\%) & $10 / 14$ & $71 \%$ & $7 / 11$ & $64 \%$ & \\
\hline Reoperation, $n$ (\%) & $3 / 14$ & $21 \%$ & $2 / 11$ & $18 \%$ & $>0.99$ \\
\hline & Median $(\boldsymbol{n}=\mathbf{1 4})$ & IQR & Median $(\boldsymbol{n}=\mathbf{1 1})$ & IQR & \\
\hline Follow-up (in years) & 13 & $9-17$ & 13 & $9-14$ & 0.69 \\
\hline PROMIS Upper Extremity & 56 & $44-56$ & 56 & $41-56$ & 0.52 \\
\hline PROMIS Pain Intensity & 39 & $39-52$ & 39 & $39-49$ & 0.80 \\
\hline QuickDASH & 2.3 & $0-23$ & 4.5 & $2.3-14$ & 0.61 \\
\hline Pain (0-10) & 1 & $0-3$ & 1 & $0-2$ & 0.40 \\
\hline Satisfaction (0-10) & 9 & $7-10$ & 10 & $8-10$ & 0.36 \\
\hline
\end{tabular}

Abbreviations: IQR, interquartile range, VBG, vascularized bone graft. 


\section{Discussion}

This study has some shortcomings. First, although the $42 \%$ response rate is comparable to other studies, ${ }^{8}$ patientreported outcomes were available for less than half of the patients. This may have introduced sampling or attrition bias. Second, this is a retrospective study of patients treated by several surgeons over a 22-year span at three hospitals. Presurgical data were not reported homogeneously in the medical record; therefore, we were unable to determine postoperative change. Third, complications and reoperations may have been underreported for patients who potentially had their follow-up elsewhere.

We observed that 6 of 48 patients had proximal row carpectomy after radial shortening osteotomy due to persistent pain. No salvage procedures were performed after combined procedures of radial shortening and vascularized bone grafting. Perhaps, additional revascularization has contributed to the lower incidence of salvage procedures, but with the numbers available we did not observe a difference. Kakinoki et al reported successful treatment of three patients with persistent pain after radial shortening osteotomy by vascularized bone grafting. ${ }^{14}$ Four patients had an additional arthroscopic debridement and no further surgeries. Previous research demonstrated that arthroscopic debridement increased wrist functional range of motion, provided excellent pain relief, and improved health-related quality of life in patients with Kienböck's disease. ${ }^{15}$ Two of 48 patients had subsequent shortening of the ulna due to new onset of symptoms consistent with ulnocarpal impaction. Our rate does not differ from the rates reported in the literature. ${ }^{8,16}$ Other complications were mostly related to hardware (e.g., removal of symptomatic hardware, extensor pollicis longus rupture, loose hardware).

We found that, in the long term, the average upper extremity physical function after surgery for Kienböck's disease (median: 56) was better than that of the general U.S. population (mean: 50) in terms of PROMIS Upper Extremity CAT. To place into context, patients with common hand conditions such as carpal tunnel syndrome (mean: 43), thumb carpometacarpal osteoarthritis (mean: 46), or trigger digit (mean: 47), have lower average PROMIS Physical Function scores at presentation. ${ }^{17}$ Furthermore, the patients in our study had low average Pain Interference scores (median: 39), mean- ing that they had little effect of pain on activities of daily life compared with patients who presented with carpal tunnel syndrome (mean: 61), trigger digit (mean: 58), thumb carpometacarpal osteoarthritis (mean: 60), or even Dupuytren disease (mean: 52). ${ }^{17}$ These findings suggest that patients with Kienböck's disease in our study adapted well after surgery.

To compare our findings with existing literature, we also administered the QuickDASH questionnaire and NRS for pain. Normative values for the DASH and QuickDASH range from 10 to 13 in the general population. ${ }^{18-20}$ The relatively low (good) average QuickDASH (median: 2.3) and pain (median: 1) scores after radial shortening are in line with those reported in the literature. Studies with more than 10 years of follow-up on average report mean (Quick)DASH scores between 6.1 and 14 and pain scores between 0.2 and 3.0 after radial shortening osteotomy ( Table 4). ${ }^{21-25}$ Luegmair et al found that patients had poorer physical function when the dominant side was affected. ${ }^{25}$ We found no such difference by hand dominance. Eleven patients in our study had a revascularization procedure performed in the same surgery. Patient-reported outcomes were comparable to those of patients who had radial shortening alone. Dehghani et al concluded from their randomized study that a combination of radial shortening and vascularized bone grafting is more efficient than shortening alone in terms of Mayo Wrist Score. ${ }^{26}$ The follow-up in that study was only 9 months. Table 4 summarizes the literature on outcome of radial shortening osteotomy for symptomatic Kienböck's disease with average follow-up duration of more than 10 years.

Most studies report improvement of pain and good patientreported physical function, but whether radial shortening alters radiographic disease progression is debated. ${ }^{27}$ Long-term pain relief and good patient-reported outcome have also been reported after nonoperative management of Kienböck's disease. Keith et $\mathrm{al}^{28}$ and Viljakka et $\mathrm{al}^{29}$ report mean DASH scores of 19 and 11, respectively, in patients with Kienböck's disease after more than 10 years on average without surgery. It is unclear whether surgery contributes to the relatively good patientreported outcomes in the literature or whether we are observing the result of the benign natural course of the pathophysiology of Kienböck's disease. Further research is needed in this area.

In conclusion, we observed that approximately one in eight patients underwent salvage surgery after radial shortening and that the overall rate of reoperation was $33 \%$. This should be taken

Table 4 Overview of the literature

\begin{tabular}{|c|c|c|c|c|c|c|c|}
\hline Author (year) & $n$ & Age & Men (\%) & $\begin{array}{l}\text { Follow-up } \\
\text { (range) }\end{array}$ & $\begin{array}{l}\text { \% Stage } \\
3 A \text { or less }\end{array}$ & (Quick)DASH (SD) & Pain (SD) \\
\hline Luegmair et al $^{25}$ & 36 & 30 & $23(64 \%)$ & $12.1(5.4-17.5)$ & $100 \%$ & $12(13)$ & $0.2(0.6)-3(2.3)^{\mathrm{a}}$ \\
\hline Raven et $\mathrm{al}^{22}$ & 9 & 31 & $5(56 \%)$ & $22(16-31)$ & $67 \%$ & $14(23.7)$ & $2.3(2.9)$ \\
\hline Rodrigues-Pinto et $\mathrm{al}^{21}$ & 18 & 32 & $13(72 \%)$ & $10.3(4-18)$ & $67 \%$ & $8.7(8)$ & - \\
\hline Viljakka et $a^{23}$ & 16 & 32 & $13(81 \%)$ & $25(20-33)$ & $88 \%$ & 6.1 & $0.9-3.0^{\mathrm{a}}$ \\
\hline Watanabe et a ${ }^{24}$ & 13 & 39 & $10(77 \%)$ & $21(14-28)$ & $77 \%$ & $8.1(8.4)$ & - \\
\hline This study & 14 & 40 & $8(57 \%)$ & $13(4-25)$ & $100 \%$ & $2.3(0-23)^{\mathrm{b}}$ & $1(0-3)^{\mathrm{b}}$ \\
\hline
\end{tabular}

Abbreviations: DASH, Disabilities of Arm, Shoulder, and Hand; SD, standard deviation.

${ }^{a}$ Pain during rest and during activity.

${ }^{\mathrm{b}}$ Median and interquartile range. 
into account when making the initial decision to treat Kienböck's disease surgically. The long-term patient-reported outcomes of radial shortening-whether or not combined with a vascularized bone graft to the lunate-for symptomatic Kienböck's disease appear to be reasonable. It is unclear whether these surgeries alter the natural history of Kienböck's disease; however, in general, these surgeries appear to yield reasonable long-term function. There appeared to be no benefit of direct revascularization in addition to radial shortening osteotomy in terms of patient-reported outcome.

\section{Ethical Approval}

This study was approved by Partners Human Research Committee, Boston, MA.

\section{Note}

This work was performed at Massachusetts General Hospital.

\section{Funding}

None.

\section{Conflict of Interest}

None declared.

\section{References}

1 Allan CH, Joshi A, Lichtman DM. Kienbock's disease: diagnosis and treatment. J Am Acad Orthop Surg 2001;9(02):128-136

2 Muramatsu K, Ihara K, Kawai S, Doi K. Ulnar variance and the role of joint levelling procedure for Kienböck's disease. Int Orthop 2003;27(04):240-243

3 Paksima N, Canedo A. Kienböck's disease. J Hand Surg Am 2009;34 (10):1886-1889

4 Danoff JR, Cuellar DO, O J, Strauch RJ. The management of Kienböck disease: a survey of the ASSH Membership. J Wrist Surg 2015;4(01):43-48

5 Kolovich GP, Kalu CM, Ruff ME. Current trends in treatment of Kienböck disease: a survey of hand surgeons. Hand (N Y) 2016;11 (01):113-118

6 Horii E, Garcia-Elias M, Bishop AT, Cooney WP, Linscheid RL, Chao EY. Effect on force transmission across the carpus in procedures used to treat Kienböck's disease. J Hand Surg Am 1990;15(03):393-400

7 Weiss AP, Weiland AJ, Moore JR, Wilgis EF. Radial shortening for Kienböck disease. J Bone Joint Surg Am 1991;73(03):384-391

8 Wintman BI, Imbriglia JE, Buterbaugh GA, Hagberg WC. Operative treatment with radial shortening in Kienböck's disease. Orthopedics $2001 ; 24(04): 365-371$

9 Iwasaki N, Minami A, Oizumi N, Yamane S, Suenaga N, Kato H. Predictors of clinical results of radial osteotomies for Kienböck's disease. Clin Orthop Relat Res 2003;(415):157-162

10 Goldfarb CA, Hsu J, Gelberman RH, Boyer MI. The Lichtman classification for Kienböck's disease: an assessment of reliability. J Hand Surg Am 2003;28(01):74-80
11 Döring AC, Nota SP, Hageman MG, Ring DC. Measurement of upper extremity disability using the Patient-Reported Outcomes Measurement Information System. J Hand Surg Am 2014;39(06): 1160-1165

12 Steyers CM, Blair WF. Measuring ulnar variance: a comparison of techniques. J Hand Surg Am 1989;14(04):607-612

13 Hung M, Tyser A, Saltzman CL, et al. Establishing the minimal clinically important difference for the PROMIS and qDASH: level 1 evidence. J Hand Surg Am 2018;43:S22

14 Kakinoki R, Yamakawa T, Nakayama K, Morimoto Y, Nakamura T. Treatment of progressive necrosis of the lunate bone (Kienböck disease) after unsuccessful radial osteotomy. Scand J Plast Reconstr Surg Hand Surg 2007;41(05):267-271

15 Menth-Chiari WA, Poehling GG, Wiesler ER, Ruch DS. Arthroscopic debridement for the treatment of Kienbock's disease. Arthroscopy 1999;15(01):12-19

16 Nakamura R, Horii E, Imaeda T. Excessive radial shortening in Kienböck's disease. J Hand Surg Br 1990;15(01):46-48

17 Beleckas CM, Gerull W, Wright M, et al. Variability of PROMIS scores across hand conditions. J Hand Surg Am 2019;44:186.e1-191.e1

18 Hunsaker FG, Cioffi DA, Amadio PC, Wright JG, Caughlin B. The American Academy of Orthopaedic Surgeons outcomes instruments: normative values from the general population. J Bone Joint Surg Am 2002;84(02):208-215

19 Jester A, Harth A, Germann G. Measuring levels of upper-extremity disability in employed adults using the DASH Questionnaire. J Hand Surg Am 2005;30:1074.e1-1074.e10

20 Aasheim T, Finsen V. The DASH and the QuickDASH instruments. Normative values in the general population in Norway. J Hand Surg Eur Vol 2014;39(02):140-144

21 Rodrigues-Pinto R, Freitas D, Costa LD, et al. Clinical and radiological results following radial osteotomy in patients with Kienböck's disease: four- to 18-year follow-up. J Bone Joint Surg $\mathrm{Br}$ 2012;94(02):222-226

22 Raven EE, Haverkamp D, Marti RK. Outcome of Kienböck's disease 22 years after distal radius shortening osteotomy. Clin Orthop Relat Res 2007;460(460):137-141

23 Viljakka T, Tallroth K, Vastamäki M. Long-term outcome (20 to 33 years) of radial shortening osteotomy for Kienböck's lunatomalacia. J Hand Surg Eur Vol 2014;39(07):761-769

24 Watanabe T, Takahara M, Tsuchida H, Yamahara S, Kikuchi N, Ogino T. Long-term follow-up of radial shortening osteotomy for Kienbock disease. J Bone Joint Surg Am 2008;90(08):1705-1711

25 Luegmair M, Goehtz F, Kalb K, Cip J, van Schoonhoven J. Radial shortening osteotomy for treatment of Lichtman Stage IIIA Kienböck disease. J Hand Surg Eur Vol 2017;42(03):253-259

26 Dehghani M, Moshgelani MA, Nouraei MH, Dehghani S, Gholshahi M. Clinical outcomes of radial shortening osteotomy and vascularized bone graft in Kienböck's disease. Int Sch Res Notices 2014; 2014:956369

27 van Leeuwen WF, Janssen SJ, Ring D. Radiographic progression of Kienböck disease: radial shortening versus no surgery. J Hand Surg Am 2016;41(06):681-688

28 Keith PP, Nuttall D, Trail I. Long-term outcome of nonsurgically managed Kienböck's disease. J Hand Surg Am 2004;29(01):63-67

29 Viljakka T, Tallroth K, Vastamäki M. Long-term natural outcome (7-26years) of Lichtman stage III Kienböck's lunatomalacia. Scand J Surg 2016;105(02):125-132 